\title{
Assessment of Induction Machine Efficiency with Comments on New Standard IEC 60034-2-1
}

\author{
Wenping Cao \\ School of Science and Technology \\ University of Teesside, United Kingdom \\ w.cao@tees.ac.uk
}

\begin{abstract}
The paper assesses the efficiency of induction machines and measurement uncertainties arising from various input-output testing methods used in industry. Existing testing standards vary in methodology, procedure and required instrumentation accuracy, thus leading to significant differences in the experimentally determined efficiency for the same induction machine tested to the different standards as well as by different testing personnel. This paper focuses on the recently published IEC standard 60034-2-1, with comparisons of its previous version (IEC 34-2), and IEEE 112-B. Five induction machines with ratings between 7.5 and $150 \mathrm{~kW}$ are carefully tested using these methods and power loss results are validated by a separate calorimeter. Through theoretical analysis of measurement uncertainty using realistic perturbation-based estimation (RPBE) on these results, IEC 60034-2-1 is assessed in terms of its effectiveness and improvements over its previous version. Attention is paid particularly to these factors significantly impacting the machine efficiency such as determinations of stray load losses (SLL), stator winding resistance, stator winding temperature, and detailed specifications of testing procedures.
\end{abstract}

\section{INTRODUCTION}

Induction machines are perhaps the dominant form of industrial drive both in terms of fixed speed and of variable speed applications. For a long time the market and thus design effort have been focused mainly on the capital costs of these machines. However, with the continual rise of energy prices and an increasing awareness of environmental conservation issues, machine efficiency is inevitably becoming a key factor when an induction machine is designed, manufactured and sold.

To produce a highly efficient machine is one thing but to put a correct efficiency figure on its nameplate is quite another. This is because the measured nominal efficiency can be significantly influenced by the testing method used, and this of course is subject to uncertainty in both the application of the method and in the experimental measurements obtained. Indeed, there can sometimes be a difference of over $3 \%$ between different testing methods [1], which represents an enormous gap if it is considered in the context of the population and duty of induction machines on a global scale. Consequently, it is important to fully appreciate these uncertainties involved in determining machine efficiency when assessing potential energy savings and when trying to fix target efficiencies by legislation [2]. Without a correct (or at least consistent) determination of machine efficiency, it is difficult to make meaningful improvements by machine design. Furthermore, marketplace competition would not operate effectively to favor those manufacturers who make more efficient machines.

Previously, the International Electrotechnical Commission (IEC) standard 34-2 [3] was widely used in Europe for determining induction machine efficiency but was criticised for several decades due to its inaccuracy and inconsistency in determining the machine efficiency, especially the way it predicted the stray load loss (SLL). The new standard IEC 60034-2-1 [4] was published in September 2007 and thought to be a refinement of IEC 34-2. However, its effectiveness has not yet been reported in the literature.

This paper investigates the key modifications made in the new IEC standard and then outlines the analytical methods to assess the machine efficiency experimentally determined by both IEC standards. By investigating the measurement uncertainties and their relative influence on the losses and efficiency, a realistic perturbation-based estimation (RPBE) method is proposed which incorporates all the significant error sources and which can be used to evaluate the overall accuracy of efficiency calculations.

A test rig is set up to directly measure the machine power loss by the standard methods and a high-precision calorimeter is employed to justify these power loss measurements. Five general purpose three-phase induction machines rated at 7.5, $30,75,110$, and $150 \mathrm{~kW}$ are carefully tested using the IEC 342, IEC 60034-2-1 and IEEE 112-B [5] methods for comparison.

The aim of this paper is to assist in interpretations of measured efficiency data and particularly to check the effectiveness of the IEC 60034-2-1.

\section{TESTING STANDARDS}

It is widely accepted that IEEE 112 represents a milestone in machine testing standards and has gained in popularity. In this standard, relatively high instrumentation accuracy is specified, as is shown in Table I. Moreover, SLL is determined directly in method B when using the dynamometer method by loss segregation and by smoothing the residual loss to fit in a linear curve when plotting residual loss versus the load torque squared. As a consequence, this standard serves as a benchmark in this paper for comparison purpose. 
Proceedings of the 2008 International Conference on Electrical Machines

TABLE I

INSTRUMENTATION ACCURACY AND EFFICIENCY ESTIMATION (\%)

\begin{tabular}{|l|c|c|c|}
\hline Variable & IEEE 112-B & IEC 34-2 & IEC 60034-2-1 \\
\hline Voltage & 0.2 & 1 & 0.2 \\
\hline Current & 0.2 & 1 & 0.2 \\
\hline Power & 0.2 & 1 & 0.2 \\
\hline Torque & 0.2 & 1 & 0.2 \\
\hline Speed & $1 \mathrm{rpm}$ & $2 \mathrm{rpm}$ & $1 \mathrm{rpm}$ \\
\hline Frequency & 0.1 & 1 & 0.1 \\
\hline Resistance & 0.2 & 0.5 & 0.2 \\
\hline Temperature & $1^{\circ} \mathrm{C}$ & $2^{\circ} \mathrm{C}$ & $1^{\circ} \mathrm{C}$ \\
\hline WCE & 0.31 & 1.3 & 0.34 \\
\hline RPBE & 0.17 & 0.72 & 0.19 \\
\hline
\end{tabular}

\section{A. IEC 34-2}

IEC 34-2 defines a set of loose instrumentation accuracy in the measurement, also shown in Table I. Contrary to IEEE $112-\mathrm{B}$ in determining the SLL, it allocates a nominal $0.5 \%$ of input power to the SLL by recognizing the experimental difficulty in measuring this loss component. Because this level of SLL is atypically low for most small motors below $150 \mathrm{~kW}$, it has been questioned by many authors [6]-[10].

In the authors' previous study [1] on 23 new induction machines rated between 5.5 and $225 \mathrm{~kW}$, the ratios of the SLL to the input power were found to be within the range of 0.1$1.8 \%$. As shown in Fig. 1, the SLL varies significantly from one machine to another even though some are similarly rated machines.

Obviously, for this group of 23 new products, the IEC 34-2 standard underestimates the magnitude of the SLL. In effect, IEC 34-2 is thought of being relatively easy with repeatable methods, which are not critically dependent on high precision measuring instruments, for comparing the efficiency of different machines. These downsides have been improved in the new standard.

\section{B. IEC 60034-2-1}

Also shown in Table I, IEC 60034-2-1 specifies the same instrumentation accuracy as the IEEE 112 for measuring the key electrical and mechanical parameters. Furthermore, it also specifies an accurate determination of iron loss where the stator resistance voltage drop is taken into account.

In determining SLL, the IEC 60034-2-1 provides two methods: one is direct determination and the other, indirect determination. In the direct method, it uses the exactly same techniques as the IEEE counterpart to segregate the residual loss and to smooth it by linear regression analysis. The indirect method allocates varying ratios to SLL, which are a function of machine ratings, as plotted in Fig. 2 for illustration. This is clearly an improvement compared to that fixed allowance made in the previous version. Nonetheless, the SLL is indeed machine specific [11], and any arbitrary allocation for this loss is unjustifiable when measurements can be made. In this paper, the focus is then placed on the direct method of IEC 60034-2-1 for comparison.

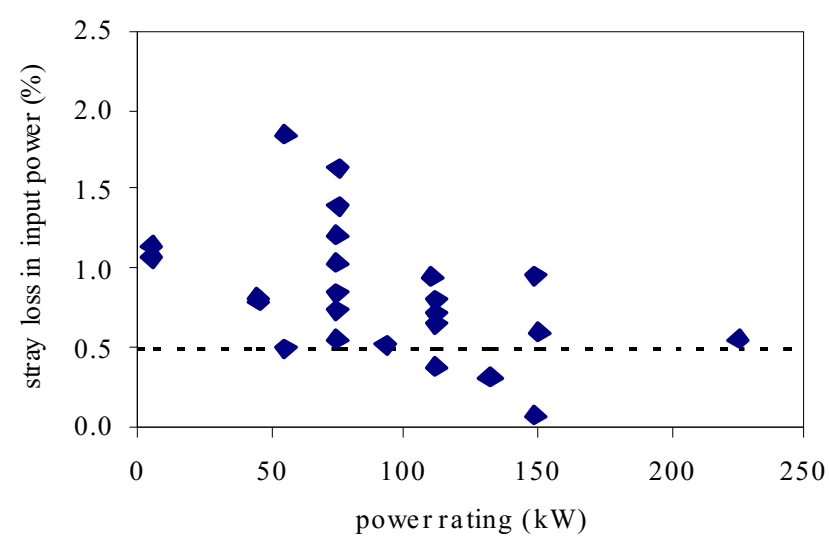

Fig. 1. The spread of the ratios of SSL to the input power

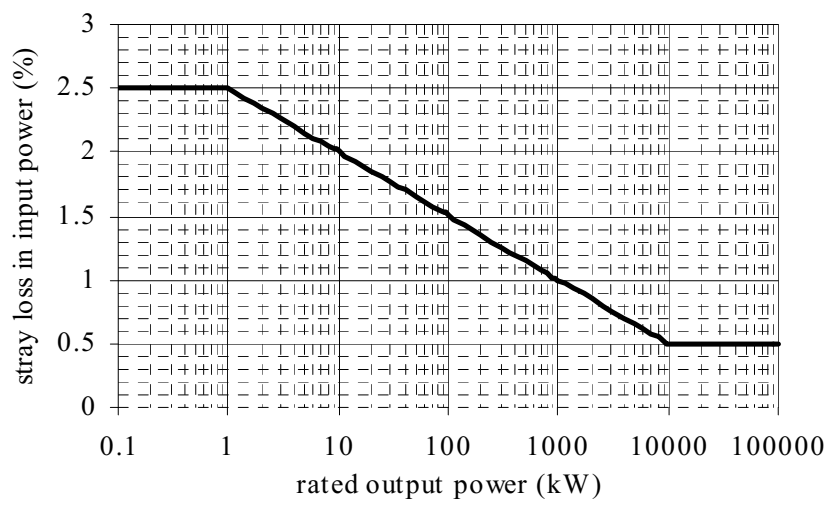

Fig. 2. Assigned allowance for SLL in IEC 60034-2-1

Additionally, in the IEC 34-2, the stator winding resistance is required to be corrected to the specified temperatures according to the machine's thermal class. But in the 60034-2-1 standard, this is corrected to an ambient temperature of $25^{\circ} \mathrm{C}$ according to its rated temperature rise, which is similar to the requirement of the IEEE 112-B.

Based on these improvements made in the new IEC standard, one might expect to have more accurate efficiency results than the old IEC standard, and similar results to the IEEE 112-B.

\section{SOURCE OF UNCERTAINTY IN EVALUATING MACHINE EFFICIENCY}

In a scientific measurement, the associated error is generally composed of three components.

$$
\xi=\xi_{m}+\xi_{h}+\xi_{i}
$$

where $\xi$ is the overall measurement error, $\xi_{m}, \xi_{h}$ and $\xi_{i}$ are the methodological, human and instrumental errors, respectively.

In machine testing, the first two uncertainty sources arise from the methodologies, testing procedures and instrumental accuracy defined by the standards. The human error is generally associated to the ways the personnel interpreting the standards, conducting the test and processing the test results. 
Although the IEC 60034-2-1 has incorporated some of the IEEE 112 standard into its specifications, it is not detailed in the testing procedures as IEEE 112 is. For instance, there is no definition about the position the temperature sensors should attach to. In fact, inserting the temperature probes into different machine position would give quite different temperature readings, especially when the machine is experiencing a high rate of temperature change. For induction machine with rating below $150 \mathrm{~kW}$, stator conductor loss is probably the single greatest loss component. In conjunction with the uncertainty in resistance measurement, the accurate determination of stator conductor loss poses a particular challenge since how to measure the two in IEC 60034-2-1 is open to interpretation.

Another source of uncertainty in IEC 60034-2-1 may be from the definitions of the testing points. Taking the load test for example, the load torque values in the standard are set at least 6 approximately equally spaced points between $25 \%$ and $150 \%$. However, when deriving the SLL the test results are plotted against torque squared. It is obvious that, by extrapolating a linear line to zero torque, the higher load points would carry a greater weighting factor over lower ones. This is shown in Fig. 3 for illustration. The similar scenario occurs for no load tests in specifying the voltage points to derive windage and friction losses, and core loss. Indeed these problems can be easily overcome by specifying the testing points with approximately equal spacings of voltage squared (for no-load tests) or torque squared (for load tests).

\section{Methods of Estimating the MeAsurement ERrors}

In the literature, the worst case estimation (WCE) [12]-[13] has been previously reported for the evaluation of measurement uncertainty. This technique is to summarize all the possible and maximum uncertainties present in a measurement with reference to the instrumental accuracy. In reality this method represents an over-exaggeration of measurement uncertainty. In order to improve this technique, a $\mathrm{RPBE}$ recognizes the differing influence and significance of

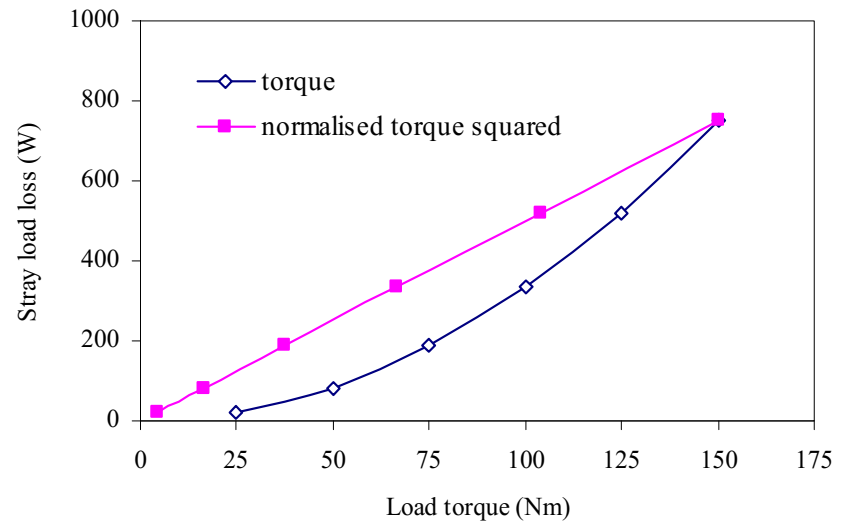

Fig. 3. Curve fitting for SLL in IEC 60034-2-1 each measured parameter, and summarizes all the major uncertainty contributors in a quadrature addition, with reference to the instrumental accuracy of these parameters defined in the standards.

When a number of instruments are involved in a measurement, a multi-variable equation can be used to represent this complex system.

$$
y=f\left(x_{i}, z_{j}\right)
$$

where $y$ is the output variable (e.g. efficiency), $x_{i}(i=1, \ldots, n)$ are the input variables and $z_{j}(j=1, \ldots, m)$ are additive noise that is not purely comprised of bias levels.

A perturbation $\Delta x$ in the independent variable $x$ will lead to a deviation $\Delta y$ in $y$. The influence coefficient of the parameter $x$ is defined as [14]:

$$
I_{x}=\frac{\Delta y / y}{\Delta x / x_{i}}=\frac{\partial f}{\partial x_{i}} \frac{x_{i}}{y}
$$

Provided all uncertainties are independent and random, the absolute error of the measurement at the output $y$ may be written as:

$$
\Delta y=y \sum_{i=1}^{n} I_{x_{i}} \frac{\Delta x_{i}}{x_{i}}+\sum_{j=1}^{m} \frac{\partial f}{\partial z_{j}} z_{j}
$$

Thus, the WCE for $y$ can be expressed as:

$$
\frac{\Delta y}{y}=\sum_{i=1}^{n} I_{x_{i}} \frac{\Delta x_{i}}{x_{i}}+\frac{1}{y} \sum_{j=1}^{m} \frac{\partial f}{\partial z_{j}} z_{j}
$$

The overall RPBE can be defined as:

$$
\frac{\Delta y}{y}=\sqrt{\sum_{i=1}^{n}\left(I_{x_{i}} \frac{\Delta x_{i}}{x_{i}}\right)^{2}+\frac{1}{y^{2}} \sum_{j=1}^{m}\left(\frac{\partial f}{\partial z_{j}} z_{j}\right)^{2}}
$$

\section{TEST FACILITIES}

Fig. 4 shows a schematic of the test rig for standard machine testing used in this study.

The test equipment consists of a dc load machine coupled to the test machine by a torque transducer mounted in a Carden shaft. There are no additional bearings between the torque transducer and the test machine. Armature current control using a Ward Leonard system ensures smooth torque from the dc machine even at light load. The ac supply to the test machine is provided by an ac generator, which is driven by an inverter-fed, synchronous motor. This configuration gives precise and constant supply frequency. The automated voltage regulator of the generator gives voltage control from 0 right through to $130 \%$ of nominal rated value. Supply imbalance and distortion are negligible with a balanced load. Coupled to the same shaft as the generator and synchronous motor is a dc machine which forms part of the Ward Leonard system and which reclaims energy from the test machine.

Apart from this test rig, a separate calorimeter is also employed for validation of the power loss measurement. 


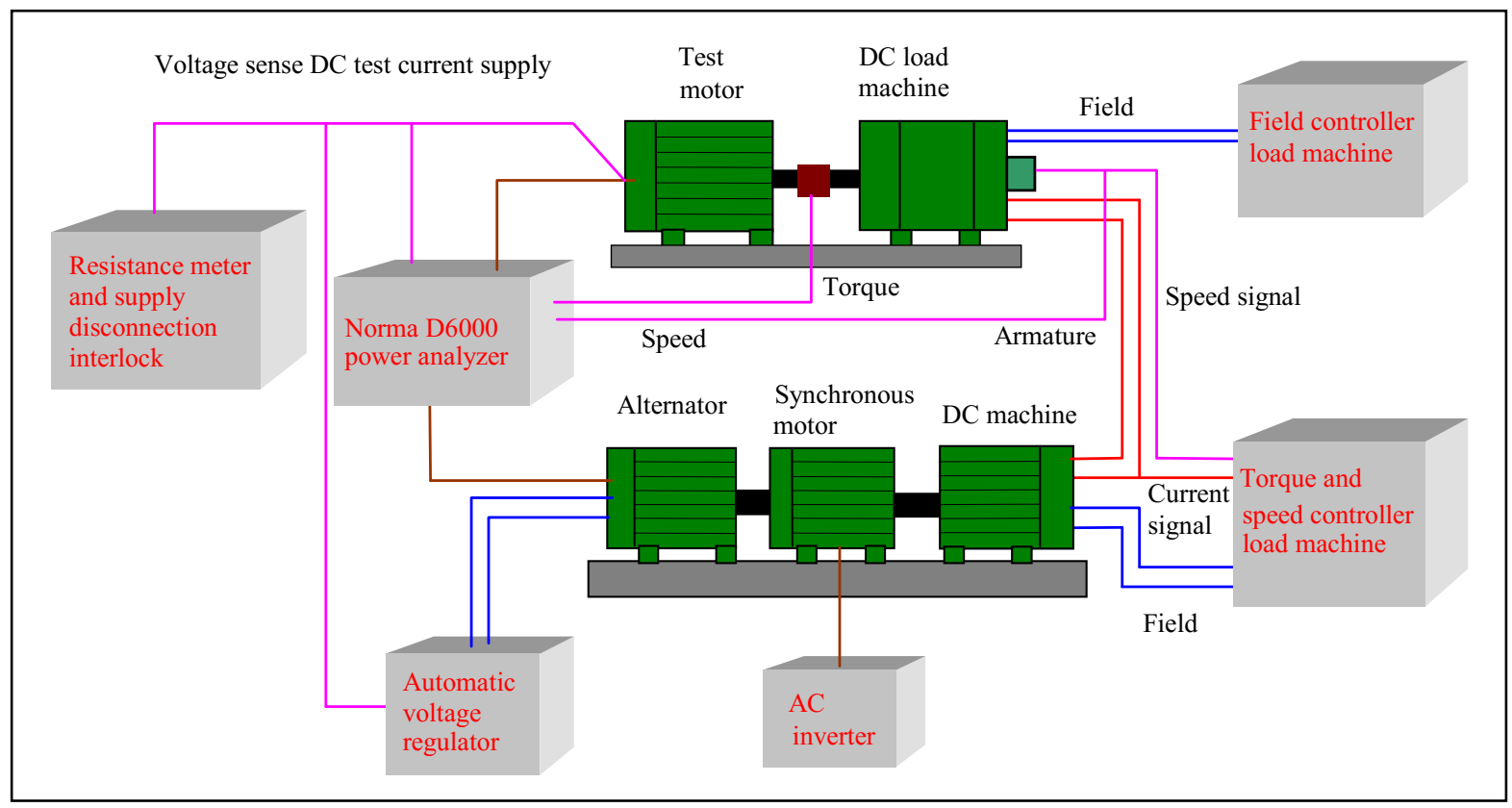

Fig. 4. Schematic of test rig used for standard machine testing

This calorimeter measures power loss directly but is limited to machines of approximately $30 \mathrm{~kW}$. Its overall accuracy is better than $0.2 \%$ of the power loss. However, the calorimetric tests are all long duration and costly. More details of this calorimeter are given in [15].

\section{RESULTS AND DISCUSSION}

Machine details are listed in Appendix. All induction machines are tested on the test rig following the standard methods defined in IEC 34-2, 60034-2-1 and IEEE 112-B, respectively. Among these five machines, a $30 \mathrm{~kW}$ machine (labeled as machine 2) is also tested within a $30 \mathrm{~kW}$ calorimeter.

The standard test procedures are based on no load tests, full load and part load tests. Part-load tests are required to be taken as quickly as possible, from the highest load to the lowest, following a steady-state rated load test. i.e., part-load tests are essentially conducted at the temperature of the machine related to the full load condition and winding temperatures are inferred by thermocouple in virtually all cases. But in the calorimetric tests, the part load tests are all at the steady state machine temperature associated with the part load condition of operation. This might bring about some differences between the two approaches, especially under those light loads.

\section{A. Calorimetric tests}

The calorimeter in this study provides an alternative means and high-precision power loss measurement. Because the SLL in the induction machine is a sensitive component derived from subtracting the identifiable losses from the total loss, it is used in this study for comparison between calorimetric and input- output methods in detecting small loss change. Loss segregation complies with IEEE 112 method B. Test results are plotted in Fig. 5 for comparison.

As shown in this figure, a range of load values including $25 \%, 50 \%, 75 \%, 90 \%$ and $100 \%$ are obtained by the calorimeter along with one set of IEEE 112-B test results which extend load points further to $125 \%$. Fig. 5 clearly shows a good agreement between the residual loss values obtained by calorimetric and IEEE 112-B methods. From the both curves it can be observed that the calorimetric results present a linear curve shape going through the zero load points whilst IEEE 112-B results give a small degree of curvature and zero offset. Again, this distortion might be caused by inappropriate tracings of stator winding temperatures by IEEE 1112-B when the machine is undertaking a rapid temperature drop from reducing loads.

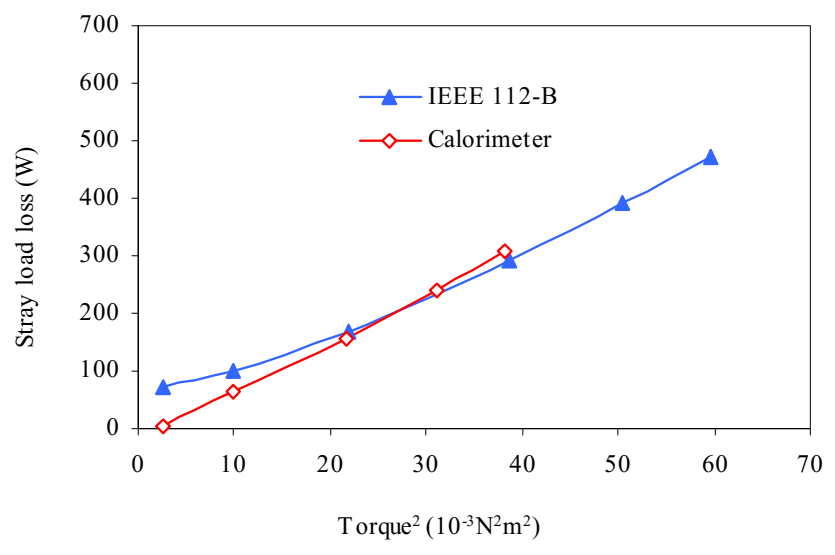

Fig. 5. Calorimeter tests for validation 
Yet, the slope of IEEE 112-B curves fitted by regression analysis is very close to that of calorimetric results. According to the IEEE standard, SLL is then corrected to fit to a specific machine model by removing the constant offset and applying linearization for SLL. The correction factor exceeds 0.95 and constant zero offset is within the accuracy the instrumentation could achieve.

\section{B. Power losses}

For standard tests on the five machines, their loss components present different types of discrepancy. Between the old and new IEC standards, the determinations of core loss, friction and windage losses are essentially the same. Although the new standard specifies an accurate determination of iron loss to take account of the stator resistance voltage drop, this only affects part-load results, not the rated load values which are the interest of this paper.

As a result, comparisons are focused on stator conductor loss, rotor conductor loss and SSL. As shown in Fig. 6, the two IEC methods present similar rotor conductor loss and slightly different stator conductor loss. However, the biggest difference lies in SLL except machine 2. For this particular machine, the actual SLL happens to be approximately $0.5 \%$ of input power so that the two methods give the same SLL. For all other four machines, the differences in the SLL are significant although this loss component is generally small relative to other loss components. In the extreme case of machine 3 , the difference in SLLs is $876 \mathrm{~W}$, remarkably higher than that in stator conductor loss $(127 \mathrm{~W})$ and that in rotor conductor loss $(2 \mathrm{~W})$. Therefore, how to determine the SLL would make the key difference between one standard and another.

\section{Machine efficiencies}

Machine efficiency results are given in Table II for these five machines under test.

There are three observations that can be made from this table. Firstly, all the efficiency figures from IEC 34-2 are greater than those of the IEEE 112-B and IEC 60034-2-1. This confirms a long-standing view that the former IEC standard

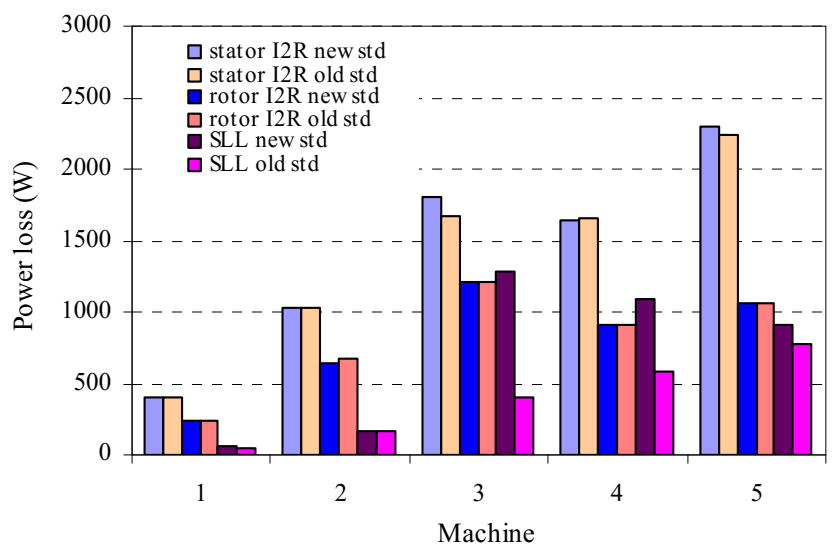

Fig. 6. Comparisons of three loss components
TABLE II

COMPARISON OF EFFICIENCIES IN \% BETWEEN THE STANDARDS

\begin{tabular}{|l|c|c|c|c|c|}
\hline Machine & $\mathbf{1}$ & $\mathbf{2}$ & $\mathbf{3}$ & $\mathbf{4}$ & $\mathbf{5}$ \\
\hline IEC old & 88.9 & 92.4 & 94.2 & 95.3 & 95.5 \\
\hline IEC new & 88.7 & 92.5 & 93.1 & 94.7 & 95.4 \\
\hline IEEE & 88.7 & 92.5 & 93 & 94.8 & 95.4 \\
\hline SSL/Pin & 0.79 & 0.51 & 1.58 & 0.94 & 0.58 \\
\hline
\end{tabular}

provides a higher efficiency value than other standards. Secondly, when the ratios of SSL to input power are investigated, these are all in excess of $0.5 \%$ for these five machines. Again, this is the main reason for the IEC efficiencies to be higher than others. Thirdly, the new IEC standard provides nearly the same efficiency figures as the IEEE counterpart. The differences in efficiency values between IEC 60034-2-1 and IEEE standards are all within the measurement accuracy. It may be said that from these results a high degree of harmonization has been achieved between the IEC 60034-2-1 and IEEE 112 standards.

\section{Uncertainty levels}

Using WCE and RPBE methods described previously, the measurement uncertainties in machine efficiency are studied in a MatLab program, including those measurement uncertainties of voltage, current, power, torque, speed, frequency, resistance, and temperature. This is done by assessing the impact of each measurement uncertainty on the machine efficiency results and by adding their significances (in efficiency values) in a quadrature manner.

The results are also given in Table I. It can be seen from it, as long as these standard methods are strictly followed, IEC 60034-2-1 is capable of determining the machine efficiency to an accuracy of $0.19 \%$ with the worst-case error of $0.34 \%$ while IEC 34 can also provide an accuracy of $0.72 \%$ with the worstcase error of $1.3 \%$. It is worth noting out that these results include instrumentation errors only. In practice, the overall measurement errors are generally greater, mainly due to human errors [16].

It is obvious that these test results justify the IEC new standard in terms of detecting a very small loss in electrical machine and providing accurate efficiency results.

\section{CONCLUSIONS}

This paper have investigated the induction machine efficiency and measurement uncertainty resulting from IEC and IEEE testing standards, with a focus placed on the recently published IEC standard 60034-2-1. The improvements of the IEC 60034-2-1 over its previous version are found in defining higher instrumentation accuracy, and particularly, more accurate method to determine the stray load loss.

The IEC 60034-2-1 is significant in providing methods of direct quantifying machine efficiency and power losses, in 
particular stray load loss. Test results from five induction machines with ratings between 7.5 and $150 \mathrm{~kW}$ have confirmed the effectiveness of this new standard. In effect, this standard has highly aligned itself with IEEE 112. The accuracy of power loss measurements by these standard methods has been validated by the calorimetric approach. The measurement uncertainty of machine efficiency has been investigated using the realistic perturbation-based estimation.

Test results also suggest that instrumentation errors alone would not be greater than $0.2 \%$ for IEC $60034-2-1$ standard. The greatest discrepancy may still lie in the human error. From the viewpoint of standard-making body, the test procedure should be defined as clear and rigid as possible. From the industry and end-users' viewpoints, education and discussion may help to minimize these sources of error.

\section{ACKNOWLEDGMENT}

The author would like to acknowledge the helpful discussions with Dr K. J. Bradley on the preparation of this paper.

\section{APPENDIX}

List OF TEST MACHINES

\begin{tabular}{|l|r|r|r|r|r|}
\hline Machine & $\mathbf{1}$ & $\mathbf{2}$ & $\mathbf{3}$ & $\mathbf{4}$ & $\mathbf{5}$ \\
\hline$k W$ & 7.5 & 30 & 75 & 110 & 150 \\
\hline Pole No. & 4 & 4 & 4 & 4 & 4 \\
\hline$H z$ & 50 & 50 & 50 & 50 & 50 \\
\hline$V$ & 400 & 400 & 400 & 400 & 400 \\
\hline$A$ & 14.5 & 54 & 142 & 198 & 255 \\
\hline$R P M$ & 1455 & 1465 & 1478 & 1487 & 1488 \\
\hline
\end{tabular}

\section{REFERENCES}

[1] W. Cao, "Accurate measurement and evaluation of losses and efficiency of new and rewound induction motors", PhD Dissertation, the University of Nottingham, September 2004
[2] US Act, The energy policy act of 1992 (EPAct)

[3] IEC 34-2: 1972 (IEC 34-2A 1974) (BS EN 60034-2), amendments 1: 1995 and amendments 2: 1996, General Requirements for Rotating Electrical Machines- Part 102: Methods for determining losses and efficiency from tests (excluding machines for traction vehicles)

[4] IEC 60034-2-1: 2007 (BS EN 60034-2-1) Rotating Electrical MachinesPart 2-1: Standard methods for determining losses and efficiency from tests (excluding machines for traction vehicles)

[5] IEEE Std 112-2004 (IEEE 112-1991, 1996), IEEE Standard Test Procedure for Polyphase Induction Motors and Generators (ANSI)

[6] E. Levi, "Polyphase motors: a direct approach to their design", John Wiley and Sons, New York, 1984

[7] C.N. Glew, "Stray load losses in induction motors: a challenge to academia", Power Engineering Journal [see also Power Engineer], Volume: 12 Issue: 1, Feb 1998 pp. 27-32

[8] H. Auinger, "Determination and designation of the efficiency of electrical machines", Power Engineering Journal [see also Power Engineer], Volume: 13 Issue: 1, Feb 1999 pp. 15-23

[9] A.I. de Almeida, F.J.T.E. Ferreira, J.F. Busch, P. Angers, "Comparative analysis of IEEE 112-B and IEC 34-2 efficiency testing standards using stray load losses in low-voltage three-phase, cage induction motors", IEEE Transactions on Industry Applications, Volume 38, Issue 2, MarchApril. 2002, pp: 608-614

[10] K.J. Bradley, W. Cao, and J. Arellano-Padilla, "Evaluation of stray load loss in induction motors with a comparison of input-output and calorimetric methods", IEEE Transactions on Energy Conversion, Volume 21, Issue 3, Sept. 2006, pp: 682-689

[11] W. Cao, K. J. Bradley and J. Allen, "Evaluation of additional loss in induction motors consequent upon repair and rewinding", IEE Proceedings on Electric Power Applications, January 2006, Volume 153, Issue 1, pp. 1-6

[12] R.S. Colby and D.L. Flora, "Measured efficiency of high efficiency and standard induction motors", Industry Applications Society Annual Meeting, 1990, Conference Record of the 1990 IEEE, 7-12 Oct. 1990, pp. $18-23$ vol. 1

[13] D.R. Turner, K.J. Binns, B.N. Shamsadeen, and D.F. Warne, "Accurate measurement of induction motor losses using balance calorimeter", IEE Proceedings, Electrical Power Applications, Vol. 138, No 5, September 1991, pp. 233-242

[14] S.G. Rabinovich, "Measurement Errors and Uncertainties: Theory and Practice", 3rd ed. New York, NY: Springer, 2005

[15] Wenping Cao, K.J. Bradley and A. Ferrah, "Development of a high precision calorimeter for measuring power loss in electrical machines", IEEE Transactions on Instrumentation and Measurement, in press

[16] Wenping Cao, K.J. Bradley, "Assessing the impacts of rewind and repeated rewinds on induction motors: is an opportunity for re-designing the machine being wasted?", IEEE Transactions on Industry Applications, Volume 42, Issue 4, July-Aug. 2006, pp: 958-964 